\title{
Fever after Percutaneous Endovascular Stent-graft in Type B Acute Aortic Dissection
}

\author{
Dan Yu, Jiangtao Yan and Hesong Zeng
}

\begin{abstract}
Background and Purpose Fever is a common symptom of acute aortic dissection. The beginning time and duration of fever after percutaneous endovascular stent-graft in patients with aortic dissection is not well known. The present study was undertaken to investigate the characteristics of fever after percutaneous endovascular stent-graft in patients with type B aortic dissection.

Methods and Results In this retrospective study the data of a total of 64 patients with type B acute aortic dissection undergoing percutaneous endovascular stent-graft were collected and analyzed. In total, 55 patients (85.9\%) had fever after stent-graft. The time of fever onset was during the initial 2 days after interventional procedure (92.7\%), and the duration of fever of most patients was not beyond 7 days after stent implantation. The fever before stent implantation was associated with the beginning time of fever after stent implantation, but not with the duration of fever.

Conclusion The results of this study could provide important reference information for the evaluation of fever before and after interventional treatment in patients with acute aortic dissection.
\end{abstract}

Key words: acute aortic dissection, percutaneous endovascular stent-graft, fever

(Intern Med 50: 2095-2098, 2011)

(DOI: 10.2169/internalmedicine.50.5733)

\section{Introduction}

Acute aortic dissection is an uncommon but potentially catastrophic disease, with 1 percent early mortality per hour if untreated (1). The Stanford classification of aortic dissection distinguishes between type A and type $\mathrm{B}$ according to the location and extent of aortic involvement. Type A indicates the dissection includes the ascending aorta, while type B dissection does not involve the ascending aorta. The development of medical and surgical therapy has dramatically improved the prognosis of acute aortic dissection (2). More recently, percutaneous endovascular stent-graft by transfemoral catheter technique has been introduced as a potential alternative to aortic repair in type B acute aortic dissection with lower procedural complications and shorter hospital stay (3).

Fever is a common symptom of acute aortic dissection (4-6). Only two papers have been published concerning the characteristics of fever associated with acute aortic dissection $(7,8)$. However, the beginning time and duration of fever after percutaneous endovascular stent-graft in patients with aortic dissection is not well known. The present study was undertaken to investigate the characteristics of fever after percutaneous endovascular stent-graft in patients with type B aortic dissection.

\section{Materials and Methods}

\section{Subjects}

This is a retrospective study. The data of patients with type B acute aortic dissection undergoing percutaneous endovascular stent-graft in Tongji Hospital of Tongji Medical College, Huazhong University of Science and Technology from 2003 to 2010 were collected in this study. All patients were diagnosed with computed tomographic arteriography and aortic angiography. The patients with a known infection

Institute of Hypertension and Department of Internal Medicine, Tongji Hospital, Tongji Medical College, Huazhong University of Science \& Technology, China

Received for publication May 1, 2011; Accepted for publication June 23, 2011

Correspondence to Dr. Jiangtao Yan, jtyan@tjh.tjmu.edu.cn 


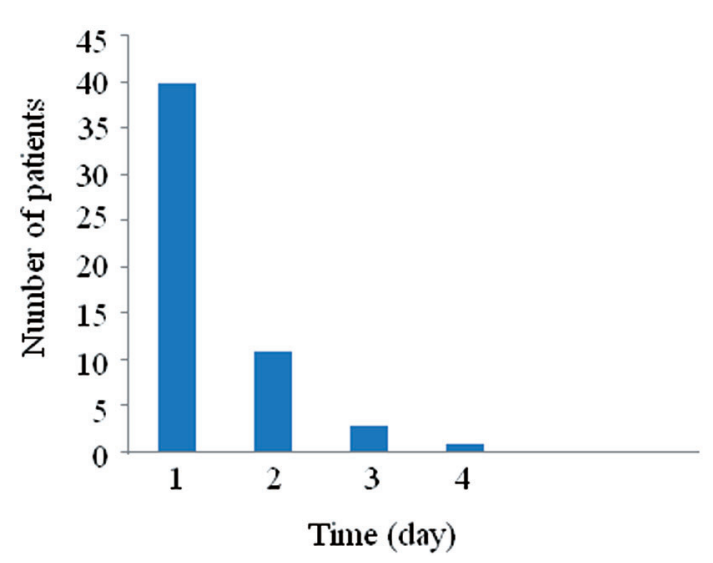

Figure 1. The onset of fever after stent implantation.

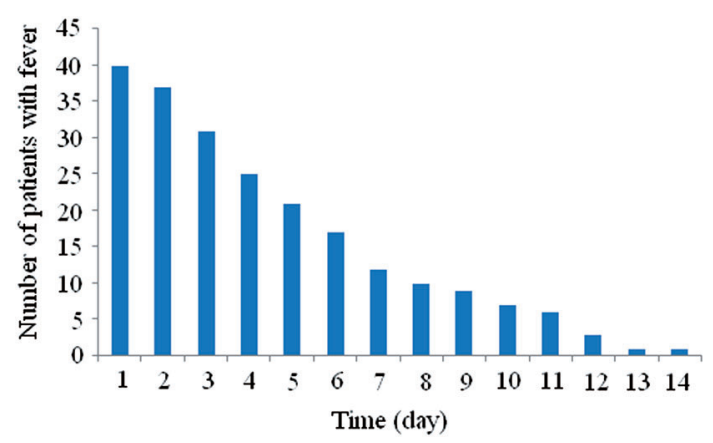

Figure 2. The duration of fever in patients who had fever onset on the first day after stent implantation.

revealed through clinical and laboratory diagnosis, aortic aneurysms, Takayasu arteritis, other collage diseases, and malignant tumor were excluded. A total of 64 subjects were selected in the present study.

\section{History-taking}

Axillary body temperatures were measured and the highest temperature during the day was considered the body temperature for the day. Fever was diagnosed when the patient's body temperature was over $37^{\circ} \mathrm{C}$.

The basic characteristics of the subjects were recorded in this study, including age, sex, history of hypertension, diabetes and smoking, fever and known infection before endovascular stent-graft, the time elapse from the occurrence of the acute aortic dissection to the endovascular stent-graft, and the lab examinations before procedure.

\section{Statistical analysis}

In this study, the analyses were performed with SPSS statistical package, version 13.0. Statistical significance for intergroup differences was analyzed by the Student's $t$ test for continuous variables and the $\chi^{2}$ test for categorical variables.

Results

\section{Characteristics of the patients}

A total of 64 type B acute aortic dissection patients who received percutaneous endovascular stent-graft were collected in this study. A total of 55 patients $(85.9 \%)$ had fever after percutaneous endovascular stent-graft, indicating that fever was a frequent symptom after stent. According to the fever after percutaneous endovascular stent-graft, the study subjects were divided into 2 groups, patients with fever and patients without fever. The characteristics of patients showed that there was no significant difference in age and sex between two groups. Two patients in the group without fever after stent implantation had fever before stent implantation, while 14 patients in the group with fever had fever before stent implantation, there was no significant difference ( $22.2 \%$ vs. $25.5 \%, \mathrm{p}>0.05)$, which suggested that there was no relationship between fever before and after stent implantation in patients with aortic dissection.

\section{The time of onset of fever after stent implantation}

Among the patients with fever after stent implantation (Fig. 1), 40 patients had fever on the first day, 11 patients had fever onset on the second day, and 3 patients on the third day, 1 patients in the fourth day, which indicated that the onset of fever generally occurred within the initial 2 days after interventional procedure $(92.7 \%)$.

\section{Duration of fever}

In order to investigate the characteristics of fever after stent implantation, we analyzed the number of patients with fever in the group who had fever on the first day and in the group who had fever onset on the second day, respectively. Figure 2 shows the number of patients with fever every day after stent implantation in the group who had fever onset on the first day, which indicated that the duration of fever in 28 patients $(70 \%)$ was not beyond 7 days, and only 1 patient (2.5\%) had a fever for over 14 days. In contrast, in the group who fever onset on the second day (Fig. 3), the duration of fever in 6 patients $(54.5 \%)$ was only 1 day, in 9 patients $(81.8 \%)$ it was not longer than 7 days, and only 1 patient had a fever for over 14 days, which suggested that the duration of fever in this group was shorter than that in the group who had fever onset on the first day.

\section{Relationship between fever before and after stent implantation}

The onset of fever according to the timing of stent implantation is (Table 1) indicated that there was no relationship between fever before and after stent implantation in patients with aortic dissection. In the group who had fever on the first day there were 12 patients who had fever on the day before stent implantation, while only 1 patient had fever before stent implantation in the group who had fever onset 


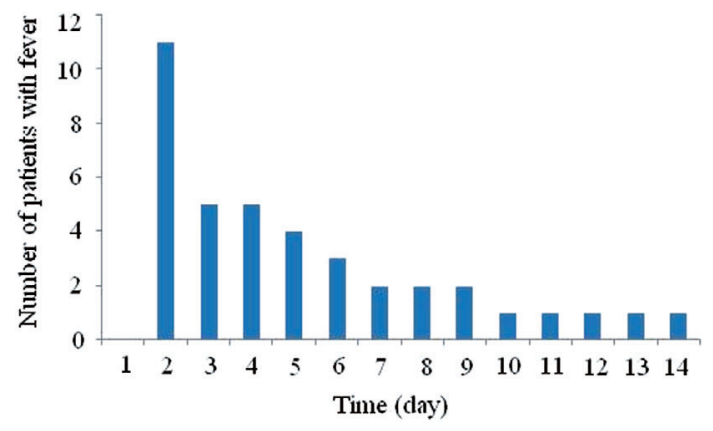

Figure 3. The duration of fever in patients who had fever onset on the second day after stent implantation.

on the second day, which suggested the fever before stent implantation was associated with the onset of fever after stent implantation. However, there was no significant difference in the duration of fever after stent implantation between patients with fever before stent implantation and those without fever before implantation $(6.8 \pm 6.2$ days vs $4.5 \pm$ 3.7 days, $\mathrm{p}=0.096$ ), which suggested that the fever before stent implantation was not associated with the duration of fever after stent implantation.

\section{Discussion}

This retrospective study of patients with type B acute aortic dissection undergoing percutaneous endovascular stentgraft demonstrated that total 55 patients $(85.9 \%)$ had fever after stent-graft. The onset of fever occurred within the initial 2 days after interventional procedure $(92.7 \%)$, and the duration of fever of most patients was not beyond 7 days after stent implantation. The fever before stent implantation was associated with the onset of fever after stent implantation, but not with the duration of fever.

Fever is a common symptom of aortic dissection (4-6). A previous report indicated that about one-third of the patients with aortic dissection had manifestation of fever (9). The present study showed that $85.9 \%$ patients with acute aortic dissection had fever after stent, in whom about one-fourth of the patients already had fever the day before stent, which suggested that stent-graft could increase the possibility of fever. The fever associated with aortic dissection involves an inflammatory reaction, which is caused by thrombi, destroyed tissue, and cytokines (7). False lumen thrombosis is the main pathophysiological feature after stent-graft in acute aorta dissection. Thrombosis in the false lumen could cause an inflammatory reaction, which could be involved in the fever after stent.

Few reports have been published concerning the characteristics of fever associated with acute aortic dissection. Garcia-Romo et al (8) defined the clinical criteria of fever due to inflammation in acute aortic dissection through a retrospective analysis of 59 patients, which demonstrated that fever occurred within the first 48 hours, and episodes of fever did not affect the patient's general clinical condition.
Table 1. Characteristics of Patients

\begin{tabular}{lccc}
\hline & $\begin{array}{c}\text { Patients without } \\
\text { fever } \\
(\mathrm{n}=9)\end{array}$ & $\begin{array}{c}\text { Patients with fever } \\
(\mathrm{n}=55)\end{array}$ & p value \\
& $56.1 \pm 9.3$ & $50.2 \pm 7.8$ & $\mathrm{NS}$ \\
\hline Age, yrs & $1(11.1)$ & $8(14.5)$ & $\mathrm{NS}$ \\
Sex (Female), $\mathrm{n}(\%)$ & $2(22.2)$ & $14(25.5)$ & $\mathrm{NS}$ \\
Fever before stent, $\mathrm{n}(\%)$ & & & \\
Time of stent & 5 & 23 & \\
$1 \mathrm{w}$ & 4 & 10 & \\
2w & & 14 & \\
4w & & 8 & \\
\hline $\mathrm{w}$ & & &
\end{tabular}

The present study showed that the fever after stent-graft in the patients with acute aortic dissection had same onset as fever secondary to the acute aortic dissection. In our study the onset of fever occurred within the initial 48 hours after interventional procedure $(92.7 \%)$, in particular, $72.7 \%$ of fever occurred within 24 hours. These results indicated that the fever before and after stent-graft might have the same mechanism, such as thrombosis and cytokines, in patients with acute aortic dissection. The different time of onset of fever after stent could be associated with the different inflammatory reaction levels. However, the implantation of stent accelerates the fever due to the mechanism. Shimada et al (7) investigated the duration of fever associated with acute aortic dissection through a retrospective study of 57 patients, who were treated medically rather than surgically and judged to be free of infection, which showed that the mean duration of fever was $15.9 \pm 11$ days. However, the result of the present study suggested that the duration of fever after stent-graft was generally shorter than 7 days. Shimada et al reported that there was a negative correlation between the false lumen reduction ratio and the duration of fever in patients with acute aortic dissection, with less reduction in false lumen size seen in patients with fever for longer periods of time. Stent-graft could cover the false lumen and accelerate the thrombosis and resolution (10), which could be the mechanism for the shorter duration of fever after stentgraft. With our current limited data and the results of Shimada et al study, we could only speculate the possibility of association of the size of false lumen and the characteristics of fever after stent-graft, which should be proved by further study.

In contrast to Stanford type A acute aortic dissections, which requires emergency surgical therapy, Stanford B are treated traditionally mainly medically rather than surgically (1). In recent decades the percutaneous endovascular stent-graft by transfemoral catheter technique has been introduced as a potential alternative to aortic repair in type B acute aortic dissection, which could reduce the risk of rupture or progressive dissection of aortic (3). However, there is a controversy as to the exact time of stent-graft after the onset of acute aortic dissection. Several factors influence the 
intervention time, such as the general condition of the patient and the risk of rupture and progressive dissection. The fever before procedure is a notable factor for normal interventional treatment, which could indicate a possibility of infection and delay the intervention. Fever is a common symptom in patients with acute aortic dissection, thus it is important to identify the influence of fever before procedure on the interventional treatment. In the present study, over $20 \%$ patients had fever before the stent-graft, and the fever before stent-graft was not associated with the occurrence and duration of fever after the stent-graft. In the present study the infection-related fever had been excluded, thus those results suggested that fever due to inflammation in patients with acute aortic dissection was not the contraindication of stent-graft. Furthermore, the patients in this study had no vascular events including operation, re-stent and death during hospitalization, thus there was no difference in short-time prognosis of vascular events between patients with fever on the first day and those on the second day after stent-graft implantation. The long-time prognosis should be investigated by a follow-up study.

In summary, this study analyzed the characteristics of fever associated with inflammation in acute aortic dissection after percutaneous endovascular stent-graft, and indicated that the beginning time of fever occurred within the initial 48 hours after interventional procedure, and the duration of fever after stent-graft was generally shorter than 7 days, the fever before stent-graft was not associated with the occurrence and duration of fever after the stent-graft. The results of this study could provide important reference information for the evaluation of fever before and after interventional treatment in patients with acute aortic dissection.

The authors state that they have no Conflict of Interest (COI).

\section{References}

1. Erbel R, Alfonso F, Boileau C, et al. Diagnosis and management of aortic dissection. Eur Heart J 22: 1642-1681, 2001.

2. Erbel R, Oelert H, Meyer J, et al. Influence of medical and surgical therapy on aortic dissection evaluated by transesophageal echocardiography. Circulation 87: 1604-1615, 1993.

3. Eggebrecht H, Lönn L, Herold U, et al. Endovascular stent-graft placement for complications of acute type B aortic dissection. Curr Opin Cardiol 20: 477-483, 2005.

4. Giladi M, Pines A, Averbuch M, Hershkoviz R, Sherez J, Levo Y. Aortic dissection manifested as fever of unknown origin. Cardiology 78: 78-80, 1991

5. Dai MS, Cheng SM. Aortic dissection presenting as fever of unknown origin. Acta Cardiol 56: 37-38, 2001.

6. Gorospe L, Sendino A, Pacheco R, Alonso A, Barbado FJ, Vazquez JJ. Chronic aortic dissection as a case of fever of unknown origin. South Med J 95: 1067-1070, 2002.

7. Shimada S, Nakamura H, Kurooka A, et al. Fever associated with acute aortic dissection. Circ J 71: 766-771, 2007.

8. Garcia-Romo E, López-Medrano F, Llovet A, Lizasoain M, San Juan R, Aguado JM. Fever due to inflammation in acute aortic dissection: description and proposals for diagnostic and therapeutic management. Rev Esp Cardiol 63: 602-606, 2010.

9. Hirst AE, Johns VJ, Kime SW. Dissecting aneurysm of the aorta: A review of 505 cases. Medicine 37: 217-279, 1958.

10. Lee KH, Won JY, Lee DY, Choi D, Shim WH, Chang BC. Elective stent-graft treatment of aortic dissections. J Endovasc Ther 11: 667-675, 2004.

\section{(C) 2011 The Japanese Society of Internal Medicine http://www.naika.or.jp/imindex.html}

